\title{
Identification of plasma miRNA biomarkers for pregnancy detection in dairy cattle
}

\author{
Hyun-Joo Lim*, Hyun Jong Kim, Ji Hwan Lee, Dong Hyun Lim, Jun Kyu Son, Eun-Tae Kim, \\ Gulwon Jang and Dong-Hyeon Kim
}

Dairy Science Division, Department of Animal Resources Development, National Institute of Animal Science, Rural Development Administration, Cheonan 31000, Korea

Received October 19, 2020

Revised March 12, 2021

Accepted March 13, 2021

\section{*Correspondence \\ Hyun-Joo Lim \\ E-mail: limhj0511@korea.kr}

ORCID

https://orcid.org/0000-0001-7059-1553
ABSTRACT A pregnancy diagnosis is an important standard for control of livestock's reproduction in paricular dairy cattle. High reproductive performance in dairy animals is a essential condition to realize of high life-time production. Pregnancy diagnosis is crucial to shortening the calving interval by enabling the farmer to identify open animals so as to treat or re-breed them at the earliest opportunity. MicroRNAs are short RNA molecules which are critically involved in regulating gene expression during both health and disease. This study is sought to establish the feasible of circulating miRNAs as biomarkers of early pregnancy in cattle. We applied Illumina small-RNA sequencing to profile miRNAs in plasma samples collected from 12 non-pregnant cows ("open" cows: samples were collected before insemination (non-pregnant state) and after pregnancy check at the indicated time points) on weeks $0,4,8,12$ and 16 . Using small RNA sequencing we identified a total of 115 miRNAs that were differentially expressed weeks 16 relative to non-pregnancy ("open" cows). Weeks 8, 12 and 16 of pregnancy commonly showed a distinct increase in circulating levels of miR-221 and miR-320a. Through genome-wide analyses we have successfully profiled plasma miRNA populations associated with pregnancy in cattle. Their application in the field of reproductive biology has opened up opportunities for research communities to look for pregnancy biomarker molecules in dairy cattle.

Keywords: dairy cattle, miRNA, pregnancy diagnosis

\section{INTRODUCTION}

Mammals should reproduce to be able to lactate. Estrus detection, artificial insemination (AI) and pregnancy diagonosis are routinely performed. Good reproductive management in dairy farms is reliant on early and precise diagnosis of pregnancy. Currently, several pregnancy diagnosis tools including the rectal palpation, ultrasonography, milk progesterone test, and preganncy-associated glycoproteins have been widely utilized. Any direct or indirect method for pregnancy diagnosis must accurately distinguish between pregnant and non-pregnant animals. Pregnancy diagnosis can identify open cows, help expect calving dates, and help producers make culling decisions. Early identification of non-pregnant dairy cattle post AI can improve reproductive performance and pregnancy rate by decreasing the interval between AI services and increasing AI service rate. Identification of animals in a herd that fails to conceive within 3 weeks after insemination would reduce economic losses. Finally it extends the 
calving interval and have contributed to the decline in profitability. Thus, new technologies to identify pregnant dairy cattle early after artificial insemination (AI) may play a key role in economically viable reproductive management decision to improve reproductive performance and profitability to commercial dairy farms.

Currently, several pregnancy diagnosis tools use molecules including pregnancy-associated glycoproteins (PAGs), protein B, DG29 and preliplantation factor (PIF). Research to develop commercial indirect mehods for pregnancy diagnosis continues because these methods are non-invasive and the tests can be marketed to and performed by dairy farmers or herd employees. We aimed at finding micro RNA (miRNAs) biomarkers by hypothesisfree, small RNA next generation sequencing (NGS). miRNAs are small non-coding RNAs molecule (16-27 nt long) that act as post-transcriptional gene regulators and play important roles in regulation of gene expression. The research for easily accessible biomarkers of several diseases and physiological condition has recently focused on circulating microRNAs (miRNA). The differentially expressed miRNAs could serve as feasible biomarkers for not only early diagnosis of pregnancy but also for various livestock health and disease (Hale et al., 2014). As miRNAs are mainly secreted into small extracellular vesicles and miRNAs have been found in numerous biofluids ranging from serum and amniotic fluid to urine and milk (Reid et al., 2011; Pohler et al., 2015). They can therefore be taken as a liquid biopsy and represent ideal molecules for the use of non-invasive biomarker of disease. (Reid et al., 2011; Buschmann et al., 2016).

MicroRNAs are expeled from cells of most tissue types in plasma membrane bound extracellular vesicles (EV), in particular exosomes. The packaging of miRNA in EVs or exosomes is important in terms of a detection standpoint as ribonuclease (RNA-ases) are unable to penetrate and breakdown the miRNA allowing them to be extracted from blood and serum (Reid et al., 2011). Exosomes and EVs play a crucial role in intercellular communication, including promotion of sperm maturation, regulation of immune function, release of miRNA for a wide array of regulatory functions, as well as other roles currently under reserach (Raposo and Stoorvogel, 2013). Plasma and whole blood have found an appropriate resource of EVderived miRNA profiles, thus offering a feasible bloodborne biomarker candidate for several disease and physi- ological condition (Häusler et al., 2010; Reid et al., 2011).

Human based disease resaerch has found significant differences in profusion of miRNAs for many cancers (Lawrie et al., 2008; Häusler et al., 2010), heart disease (Tijsen et al., 2010) and sepsis (Wang et al., 2010). Furthermore, earlier studies in humans have shown that circulating miRNA profiles related to pregnancy become more pronounced as pregnancy progresses. Circulating miRNAs in maternal serum have been regarded as feasible biomarkers of pregnancy condition due to their significant impact on gene expression and regulation (Chim et al., 2008). A study by Gilad et al. (2008) identified miRNAs that are increased in profusion in pregnant humans but not in non-pregnant females. This finding led to the sharp progress of identifying miRNAs that were unique to pregnancy and across various species, although none have been thoroughly explained.

This thesis is aimed to focus on physiological role of miRNAs during pregnancy, alsso emphasizing their feasible for being biomarkers for pregnancy detection. The object of the present study was to determine the expression pattern of circulatory miRNAs in plasma of pregnant and non-pregnant dairy cows.

\section{MATERIALS AND METHODS}

\section{Animals selection and sampling}

The selected animals and the experimental protocol were approved by institutional animal ethical committee of the National Institute of Animal Science (NIAS). A total of 30 dairy cow were used according to their health condition, parity $(\geq 2)$ and with a BCS of approximately 3.5. After pregnancy diagnosis, non-pregnant cows were excluded from the analysis. For the present investigation, the blood samples were collected from individual animal (n = 12) on different weeks of pregnancy $(0,4,8,12$ and 16 weeks). Day 0 represents the control (collection of blood before artificial insemination: AI). Following AI, blood was collected from the cows till the 16 weeks of pregnancy. Briefly, about $10 \mathrm{~mL}$ whole blood was collected in EDTA tubes, and then the samples were incubated at room temperature for $1 \mathrm{~h}$. The collected blood sample was centrifuged at 3,000 rpm for $15 \mathrm{~min}$ at $4{ }^{\circ} \mathrm{C}$ to obtain plasma. After this step, the circulating cell-free nucleic acid was in the supernatant (plasma) and then the obtained plasma was transferred into a fresh $2.0 \mathrm{~mL}$ tube and the plasma samples were stored at $-80^{\circ} \mathrm{C}$ until further processing. 


\section{Confirmation of pregnancy}

Pregnancy was diagnosed by palpation per rectum of the uterine contents between days 50 and 60 after AI or ultrasonic examination to determine pregnancy status. Ultrasonography was carried out using a B-Mode ultrasound scanner (MyLab ${ }^{\mathrm{TM}}$ OneVET, esaote) equipped with a $5.0-\mathrm{MHz}$ linear array probe. Pregnancy diagnosis was confirmed by observation of embryocoele and allantoic fluid (Abdullah et al., 2014). The ovaries were also scanned for the presence of corpus luteum.

\section{Total RNA isolation}

Before RNA isolation, the exosomes in plasma were firstly isolated by using Total Exosomes Isolation kit (Invitrogen, Carlsbad, CA). Exosomal total RNA, including mRNA and miRNA, was isolated by using miRNeasy Mini Kit (Qiagen) following manufacturer's protocos. The concentration of RNA was measured by using the Qubit microRNA assay kit (Invitrogen, Carlsbad, CA). RNA integrity of all RNA eluates was assessed. And high quality (RNA integrity number $\geq 7$ ) samples from isolated total RNA were selected. The total RNA with lowest quality was not used for further study. The RNA samples were stored at $-80^{\circ} \mathrm{C}$ until further processing.

\section{Illumina sequencing and data analysis}

Total RNA $(5 \mu \mathrm{L})$ from each sample was used to construct miRNA library by using the NEXTflex Small RNA Sequencing Kit (Illumina, San Diego, CA) according to the manufacturer's instruction. Small RNA libraries were then pooled together in equal volumes for gel purification. The pooled library was sequenced by using the HiSeq 2500 system (Illumina) as 50 bp single reads. Read quality (adaptor removal, and size selection) was assessed using FastQC v0.11.5 (http://www.bioinformatics.babraham. ac.uk/projects/fastqc/) and cutadapt (Martin, 2011). The sequences with read length larger than 18 nucleotides (nt) were aligned against bovine miRNA database (miR-Base, release version 21) with the default parameters to identify known miRNAs using Bowtie2 (Langmead and Salzberg, 2012). Each library was processed separately. The expression level of miRNAs in each library was estimated by sRNAbench, which normalized reads count number of each miRNA reads per million (RPM) by the following foumula: $\mathrm{RPM}=$ (miRNA reads number/total mapped reads per library) $\times 1,000,000$. The differentially expressed $(D E)$ miRNAs were investigated by using bioinformatics tool edgeR v3.10.2 (Robinson et al., 2010). The DE miRNAs were determined by Log2 fold change (FC) $>1$ OR $<-1$ and false discovery rate (FDR) $<0.05$ based on counting reads by using HTSeq (Anders et al., 2015).

\section{Identification of novel miRNA prediction}

Prediction of novel miRNAs was performed by using the miRDeep2 software. Briefly, quality-trimmed reads from all samples were combined into a single reads file followed by the preprocessing, mapping, and novel miRNA prediction steps through mapper.pl and miRDeep2.pl scripts. Next, the FASTA files of predicted novel miRNA and quantifier.pl script were used to determine the readcounts/expression values for each novel miRNA from each sample. Combined count matrix for novel miRNAs was generated by using the custom scripts. Differential expression between control and pregnant groups was calculated by using the DESeq2 package (version 1.12.4).

\section{RESULTS}

We sequenced a total of 60 blood samples from nonpregnancy and 16 weeks of pregnancy. On average, the high throughput Illumina sequencing resulted in 22.4 million raw reads per sample from the exosomes of all stages (Table 1). $71.5 \%$ reads were uniquely mapped to annotate miRNAs in bovine genome.

Table 1. Sequencing and miRNA profiling statistics of normal and days of pregnancy samples

\begin{tabular}{|c|c|c|c|c|c|c|c|}
\hline Sample & Total reads & $\begin{array}{l}\text { Mapped } \\
\text { reads (\%) }\end{array}$ & $\begin{array}{l}\text { Precursor miRNA } \\
\text { reads }\end{array}$ & $\begin{array}{l}\text { Mature miRNA } \\
\text { reads }\end{array}$ & $\begin{array}{c}\text { Known precursor } \\
\text { with } \geq 5 \times \text { coverage }\end{array}$ & $\begin{array}{l}\text { No. known } \\
\text { miRNA }\end{array}$ & $\begin{array}{l}\text { No. novel } \\
\text { miRNA }\end{array}$ \\
\hline Normal & $24773796.0 \pm 3962727.1$ & $73.2 \pm 5.6$ & $305494.7 \pm 81383.0$ & $7713350.2 \pm 2613944.7$ & $361.7 \pm 45.0$ & $334.8 \pm 27.4$ & $491.8 \pm 138.4$ \\
\hline 8 weeks & $24122975.9 \pm 4669481.7$ & $70.7 \pm 12.5$ & $239792.4 \pm 154469.7$ & $6806040.9 \pm 3069368.0$ & $383.9 \pm 53.6$ & $314.4 \pm 43.0$ & $410.0 \pm 179.6$ \\
\hline 12 weeks & $22139499.4 \pm 3132885.2$ & $68.8 \pm 5.8$ & $155846.3 \pm 56288.9$ & $7785174.4 \pm 1930464.7$ & $374.7 \pm 28.2$ & $306.1 \pm 19.7$ & $320.7 \pm 72.9$ \\
\hline 16 weeks & $21845616.1 \pm 2814855.6$ & $70.5 \pm 6.8$ & $140514.6 \pm 54922.5$ & $7386644.1 \pm 1905019.6$ & $343.1 \pm 39.2$ & $307.0 \pm 35.0$ & $316.7 \pm 83.6$ \\
\hline
\end{tabular}

The average data of all five analyzed samples for each animal is displayed. 
Venn diagrams were used to demonstrate the overlap between pregnant groups (Fig. 1). Pregnant 8, 12 and 16 weeks groups had 2 common miRNAs (bta-miR-221 and bta-miR-320a).

To determine the miRNA expression patterns in blood samples, miRNA microarray analysis was conducted. By using small RNA sequencing we identified a total of 115 miRNAs that were differentially expressed on pregnancy compared to non-pregnancy. A total of 115 miRNAs were identified to be significantly differentially expressed between the groups; 91 miRNAs were upregulated and 26 miRNAs were downregulated (Table 2). And 91 upregulated and 26 downregulated miRNAs are listed (Table 3).

Gene ontology (GO) and pathway enrichment analysis were used to explore the functions of differentially expressed genes in bta-miR-221 (Fig. 2A). The target genes were enriched in a total of $167 \mathrm{GO}$ terms, which included 12 molecular function (GO:MF), 132 biological process (GO:BP), and 23 cellular component (GO:CC) terms, in addition to reactomes (REAC), and WiKiPathways (WP). The target genes of differentially expressed bta-miR320 a were significantly enriched in a total of 144 GO terms, which included 23 molecular function (GO:MF), 98 biological process (GO:BP), and 23 cellular component (GO:CC) terms, in addition to reactomes (REAC), and WiKiPathways (WP).

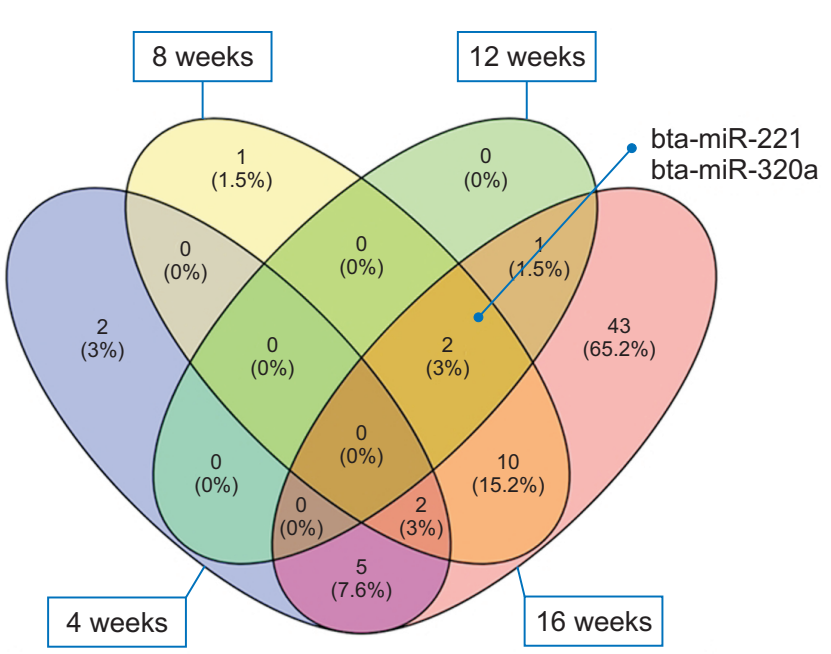

Fig. 1. Venn diagrma showing the overlap of the number and percentage of miRNAs detected in plasma samples.

\section{DISCUSSION}

This study aimed to characterize the earliest changes in miRNA expression for the purpose of define a marker for early pregnancy detection. We determined the expression pattern of circulatory miRNAs in plasma of pregnant and non-pregnant dairy cattle. Varing expression patterns of circulating miRNAs in the regulation of pregnancy has been determined in bovines (Cai et al., 2017). Many researchers are now investigating miRNAs as biomarkers for pregnancy diagnosis in the cow. There is increasing evidence that pregnancy specific miRNAs exist and may be feasible markers for pregnancy diagnosis. In 2015, exosomal miRNAs were reported to be differentially expressed in pregnant versus non-pregnant dairy cattle and dairy cattle undergoing early embryonic mortality (Pohler et al., 2015). A recent study by Fiandanese et al. (2016) identified a feasible miRNA, bta-miR 140, as an early biomarker for pregnancy diagnosis in high producing dairy cows. At day 19, bta-miR 140 was up regulated in all pregnant dairy cattle, and at day 13 onwards, it was upregulated in pregnant, non-lactating dairy cattle (Fiandanese et al., 2016). Similarly, Ioannidis and Donadeu (2016) proved different stages of the estrous cycle (day 16: bta-miR-26a, bta-miR-29c, bta-miR-138, bta-miR-204. Day 24: bta-miR-1249, day 16 \& 24: hsa-miR-4532) that were differentially expressed in pregnant heifers and miR26 a was differentially upregulated on Day 16 pregnant relative to non-pregnant heifers. The expression pattern of miR-496 and miR-125a has significantly varied during formation of bovine conceptus. This clearly suggests the role of these miRNAs in maternal-to-zygotic transcription translation (Tesfaye et al., 2009). Likewise, various miRNAs including miR-27a and miR-92b are differentially expressed during the formation of the placenta ( $\mathrm{Su}$ et al., 2010). In the present study, we report the results of circulating bta-miR-221 and bta-miR-320a were notably expressed at over 8 weeks of pregnancy. The expres-

Table 2. Differential miRNA profile expression between normal and weeks of prengnancy groups ( $p$-value $<0.05)$

\begin{tabular}{crc}
\hline Differentially expressed miRNAs & Up & Down \\
\hline 4 weeks & 9 & 8 \\
8 weeks & 16 & 8 \\
12 weeks & 3 & 2 \\
16 weeks & 63 & 8 \\
\hline
\end{tabular}


Lim et al. miRNA biomarkers for detection of pregnancy

Table 3. List of significantly differentially expressed plasma circulatory miRNAs in pregnant cows

\begin{tabular}{|c|c|c|c|c|}
\hline Week & Gene_id & a.value & p.value & q.value \\
\hline \multicolumn{5}{|l|}{ Up-regulation } \\
\hline \multirow[t]{7}{*}{4 weeks } & bta-miR-455-5p & 1.07186493 & 0.04259614 & 0.98472459 \\
\hline & bta-miR-133a & 9.90982635 & 0.03399751 & 0.98472459 \\
\hline & bta-miR-296-5p & 11.135414 & 0.01974089 & 0.98472459 \\
\hline & bta-miR-1307 & 11.6237296 & 0.02810407 & 0.98472459 \\
\hline & bta-miR-150 & 16.0778023 & 0.00042426 & 0.20576638 \\
\hline & bta-miR-339a & 16.2270717 & 0.00568494 & 0.6892985 \\
\hline & bta-miR-339b & 16.5113771 & 0.00992402 & 0.80219133 \\
\hline \multirow[t]{12}{*}{8 weeks } & bta-miR-2349 & 5.52978713 & 0.03643007 & 0.80182568 \\
\hline & bta-miR-2299-3p & 10.9842492 & 0.03782667 & 0.80182568 \\
\hline & bta-miR-2284z & 13.62152 & 0.04436674 & 0.80182568 \\
\hline & bta-miR-2284aa & 14.0033362 & 0.03916645 & 0.80182568 \\
\hline & bta-miR-451 & 14.588654 & 0.04087686 & 0.80182568 \\
\hline & bta-miR-328 & 14.599698 & 0.02207844 & 0.78481392 \\
\hline & bta-miR-375 & 15.1485213 & 0.00697631 & 0.6188333 \\
\hline & bta-miR-339a & 16.2840769 & 0.00289751 & 0.57394257 \\
\hline & bta-miR-150 & 16.3453427 & 0.01200376 & 0.78481392 \\
\hline & bta-miR-150 & 16.5251687 & 0.00168736 & 0.57394257 \\
\hline & bta-miR-221 & 17.0721993 & 0.00767149 & 0.6188333 \\
\hline & bta-miR-320a & 18.4741794 & 0.0035575 & 0.57394257 \\
\hline \multirow{18}{*}{16 weeks } & bta-miR-127 & 5.97836278 & 0.04310756 & 0.32562789 \\
\hline & bta-miR-1584-3p & 7.20287377 & 0.04827621 & 0.34076361 \\
\hline & bta-miR-6119-5p & 7.20686333 & 0.04194146 & 0.32562789 \\
\hline & bta-miR-146a & 7.34224067 & 0.02060563 & 0.24081834 \\
\hline & bta-miR-23b-5p & 7.50414778 & 0.010668 & 0.16368713 \\
\hline & bta-miR-200b & 7.55021804 & 0.00922451 & 0.15405228 \\
\hline & bta-miR-1296 & 8.48194252 & 0.02129413 & 0.24081834 \\
\hline & bta-miR-133a & 8.68207222 & 0.00108619 & 0.04102443 \\
\hline & bta-miR-211 & 8.93880225 & 0.04927539 & 0.34076361 \\
\hline & bta-miR-2284w & 9.14777305 & 0.02051713 & 0.24081834 \\
\hline & bta-miR-744 & 9.22386786 & 0.00669801 & 0.12180462 \\
\hline & bta-miR-769 & 9.79008083 & 0.00627157 & 0.12144622 \\
\hline & bta-miR-193b & 9.7992275 & 0.00941256 & 0.15405228 \\
\hline & bta-miR-2284ab & 10.7000489 & 0.03586982 & 0.29850986 \\
\hline & bta-miR-32 & 10.7206389 & 0.03160388 & 0.28213644 \\
\hline & bta-miR-2299-3p & 10.7875179 & 0.00237651 & 0.0648259 \\
\hline & bta-miR-296-5p & 10.8219527 & 0.00012621 & 0.00834593 \\
\hline & bta-miR-29b & 11.114811 & 0.00184855 & 0.06050908 \\
\hline
\end{tabular}


J Anim Reprod Biotechnol Vol. 36, No. 1, March 2021

Table 3. Continued

\begin{tabular}{|c|c|c|c|c|}
\hline Week & Gene_id & a.value & p.value & q.value \\
\hline \multirow[t]{42}{*}{16 weeks } & bta-miR-574 & 11.1383229 & 0.03148469 & 0.28213644 \\
\hline & bta-miR-877 & 11.2242015 & 0.00229709 & 0.0648259 \\
\hline & bta-miR-1307 & 11.3036664 & 0.00015298 & 0.00834593 \\
\hline & bta-miR-345-3p & 11.3975095 & 0.03964558 & 0.3191144 \\
\hline & bta-miR-2285f & 11.4813384 & 0.00310742 & 0.06646495 \\
\hline & bta-miR-98 & 11.7713234 & 0.03485827 & 0.29850986 \\
\hline & bta-miR-4286 & 11.7812685 & 0.00084086 & 0.03440499 \\
\hline & bta-miR-16a & 12.0324417 & 0.00028575 & 0.01403053 \\
\hline & bta-miR-126-3p & 12.034786 & 0.00739763 & 0.12972271 \\
\hline & bta-miR-425-3p & 12.1519812 & 0.01700118 & 0.22677729 \\
\hline & bta-miR-885 & 12.1930632 & 0.02627789 & 0.27452004 \\
\hline & bta-miR-326 & 12.3306069 & 0.0488033 & 0.34076361 \\
\hline & bta-miR-185 & 12.3441055 & 0.03792451 & 0.31034894 \\
\hline & bta-miR-378 & 12.4227355 & 0.04301751 & 0.32562789 \\
\hline & bta-miR-2285k & 12.7770755 & 0.03126496 & 0.28213644 \\
\hline & bta-miR-16b & 13.0378558 & 0.00146122 & 0.05124697 \\
\hline & bta-miR-652 & 13.1575626 & 0.02967302 & 0.28213644 \\
\hline & bta-miR-26b & 13.2076922 & 0.01328624 & 0.18851546 \\
\hline & bta-miR-2284y & 13.3654816 & 0.02049432 & 0.24081834 \\
\hline & bta-miR-143 & 13.3976177 & 0.00116375 & 0.00399116 \\
\hline & bta-miR-2284z & 13.4231816 & 0.01864053 & 0.24081834 \\
\hline & bta-miR-2284aa & 13.8486383 & 0.02880073 & 0.28213644 \\
\hline & bta-miR-874 & 13.9459277 & 0.00197643 & 0.00399116 \\
\hline & bta-miR-27a-3p & 13.9867105 & 0.00388976 & 0.07957799 \\
\hline & bta-miR-10b & 14.2690092 & 0.00977644 & 0.15484621 \\
\hline & bta-miR-215 & 14.3511691 & 0.02158046 & 0.24081834 \\
\hline & bta-miR-328 & 14.4005258 & 0.01343797 & 0.18851546 \\
\hline & bta-miR-197 & 14.4655493 & 0.02483939 & 0.27102538 \\
\hline & bta-miR-1306 & 14.5382078 & 0.02137329 & 0.24081834 \\
\hline & bta-miR-29a & 14.9644205 & 0.00311343 & 0.06646495 \\
\hline & bta-miR-375 & 15.0306074 & 0.0027077 & 0.06646495 \\
\hline & bta-miR-26a & 15.1268421 & 0.00202395 & 0.06210982 \\
\hline & bta-miR-451 & 15.1997736 & 0.00037921 & 0.01692671 \\
\hline & bta-miR-142-5p & 15.3504092 & 0.00015229 & 0.00834593 \\
\hline & bta-let-7g & 15.6507868 & 0.01342206 & 0.18851546 \\
\hline & bta-miR-339a & 15.9390783 & 0.00090517 & 0.00142078 \\
\hline & bta-miR-30e-5p & 15.9534442 & 0.0342503 & 0.29850986 \\
\hline & bta-miR-150 & 16.1951035 & 0.00254583 & 0.06578964 \\
\hline & bta-let-7b & 16.2247572 & 0.04634703 & 0.33964761 \\
\hline & bta-miR-339b & 16.230831 & 0.00197238 & 0.00399116 \\
\hline & bta-miR-221 & 16.7451505 & 0.00136249 & 0.00142078 \\
\hline & bta-miR-320a & 18.1878667 & 0.00135029 & 0.00399116 \\
\hline \multicolumn{5}{|l|}{ Down-regulation } \\
\hline \multirow[t]{8}{*}{4 weeks } & bta-miR-2454-5p & -4.28939694 & 0.00150232 & 0.36431335 \\
\hline & bta-miR-2415-5p & -4.28939694 & 0.0077144 & 0.74829656 \\
\hline & bta-miR-7861 & -4.28939694 & 0.02419468 & 0.98472459 \\
\hline & bta-miR-196a & -4.28939694 & 0.02515021 & 0.98472459 \\
\hline & bta-miR-4449 & -4.28939694 & 0.03433801 & 0.98472459 \\
\hline & bta-miR-338 & -4.28939694 & 0.03612927 & 0.98472459 \\
\hline & bta-miR-1277 & -4.28939694 & 0.03627568 & 0.98472459 \\
\hline & bta-miR-17-3p & -4.28939694 & 0.04710053 & 0.98472459 \\
\hline
\end{tabular}


Table 3. Continued

\begin{tabular}{|c|c|c|c|c|}
\hline Week & Gene_id & a.value & p.value & q.value \\
\hline \multirow[t]{8}{*}{8 weeks } & bta-miR-2313-5p & -4.48412073 & 0.00678968 & 0.6188333 \\
\hline & bta-miR-4449 & -4.48412073 & 0.01430248 & 0.78481392 \\
\hline & bta-miR-2284h-3p & -4.48412073 & 0.01739699 & 0.78481392 \\
\hline & bta-miR-2378 & -4.48412073 & 0.01801676 & 0.78481392 \\
\hline & bta-miR-154b & -4.48412073 & 0.02270123 & 0.78481392 \\
\hline & bta-miR-381 & -4.48412073 & 0.03092379 & 0.80182568 \\
\hline & bta-miR-187 & -4.48412073 & 0.03258187 & 0.80182568 \\
\hline & bta-miR-99a-3p & -4.48412073 & 0.04675656 & 0.80182568 \\
\hline \multirow[t]{2}{*}{12 weeks } & bta-miR-346 & -4.17303705 & 0.03826548 & 1 \\
\hline & bta-miR-187 & -4.17303705 & 0.0468653 & 1 \\
\hline \multirow[t]{8}{*}{16 weeks } & bta-miR-196a & -4.24490661 & 0.00643096 & 0.12144622 \\
\hline & bta-miR-2377 & -4.24490661 & 0.01708912 & 0.22677729 \\
\hline & bta-miR-2378 & -4.24490661 & 0.02605244 & 0.27452004 \\
\hline & bta-miR-2446 & -4.24490661 & 0.02746247 & 0.2809182 \\
\hline & bta-miR-346 & -4.24490661 & 0.0304451 & 0.28213644 \\
\hline & bta-miR-4449 & -4.24490661 & 0.03566852 & 0.29850986 \\
\hline & bta-miR-188 & -4.24490661 & 0.04480545 & 0.33332536 \\
\hline & bta-miR-592 & -4.24490661 & 0.04783157 & 0.34076361 \\
\hline
\end{tabular}

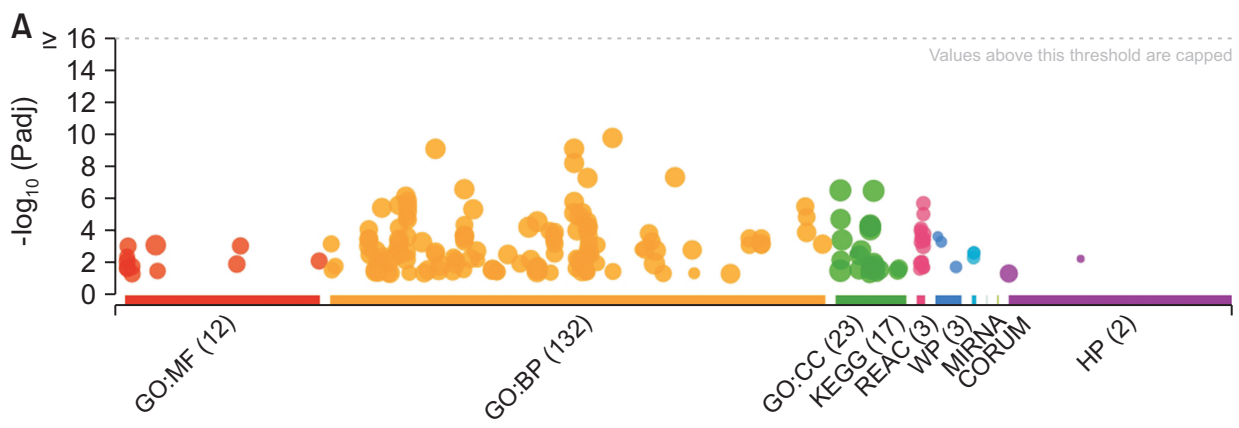

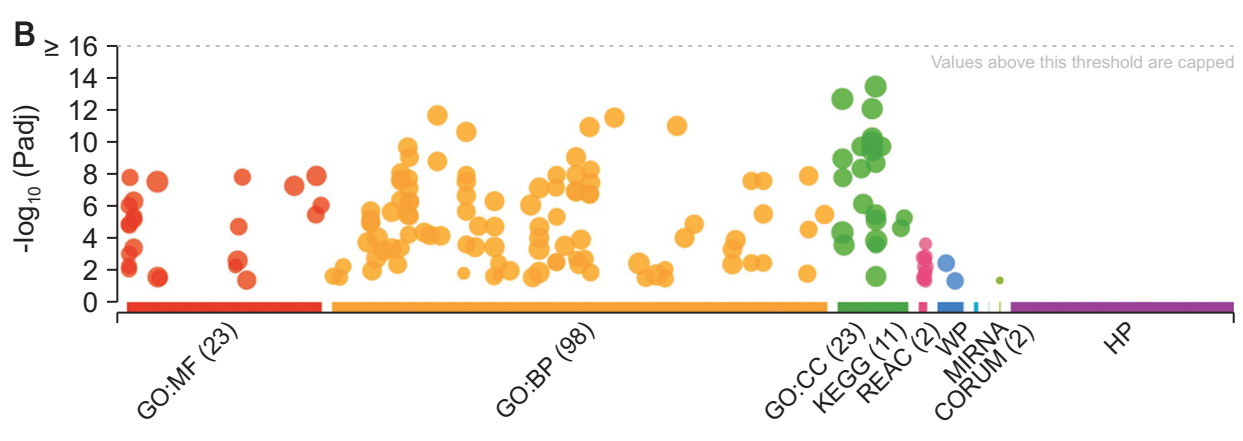

Fig. 2. Manhattan plot illustrating the differentially expressed gene-enriched GO terms (MF, molecular function; $\mathrm{BP}$, biological process; and $\mathrm{CC}$, cellular component) and KEGG pathways across reactome pathways (REAC), WiKiPathways (WP), transcription factor (TF), microRNA target base (MIRNA), and human phenotype ontology (HP) term categories. (A) bta-miR-221 enriched in $\mathrm{GO}$ terms and pathways. (B) bta-miR-320a enriched in GO terms and pathways. sion of miR-221 during pregnancy indicates the interface between maternal and fetal. Also miR-320 may promote uterine migration of endometrial stromal cells during gestation (Bidarimath et al., 2014).

Increading proofes indicated the presence of human placenta-specific miRNAs in maternal circulation. The expression of placenta-specific human chromosome 19
miRNA cluster (C19MC); hsa-miR-515-3p, hsa-miR-517a, hsa-miR-517c, hsa-miR-518b, and hsa-miR-526b increased in maternal circulation during the third trimester of pregnancy and decreased after parturition (Kotlabova et al., 2011). The villous of trophoblast cells shed the C19MC miRNA cluster encapsulated in exosomes and could be the main source of placenta-specific miRNAs 
in maternal circulation (Luo et al., 2009; Donker et al., 2012). Furthermore, according to Morales-Prieto et al. (2013) the human chromosome 14 miRNA cluster (C14MC) is also announced to be related to pregnancy. Other miRNAs involving miR-141, miR-149, miR-299-5p, and miR135 , which are offered to be aplenty expressed in placenta were also augmented in plasma of pregnant women and their concentration declines after parturition (Chim et al., 2008). Eariler study expressed that 25 miRNAs were differentially expressed between exosomes of maternal serum derived from non-pregnant and day 30 and 90 pregnant ewes (Cleys et al., 2014).

Even though purification is essential to accuarate optimal miRNA for pregnancy detection, results mean that miRNAs have feasible as an early pregnancy diagnosis tool. In addition, miRNA may offer information to represent embryonic viability. A study indicate dairy cattle that go through embryo mortality compared to dairy cattle that have a successful pregnancy have a significantly rose abundance of unique miRNAs at days 17 and 24 of conception (Reese et al., 2016). Future studies are needed to evaluate the repeatability of these result and to decide unique miRNA most appropriate for embryo viability survey.

Two new and generally available technologies for reproductive management include hormonal protocols such as Ovsynch (Pursley et al., 1995, 1997) and Presynch/ Ovsynch (Moreira et al., 2001; Navanukraw et al., 2004) that synchronize ovulation and allow for TAI, and use of transrectal ultrasonography for early detection of nonpregnant cows. Dairy farms must schedule and administer artificial inseminations, hormone injections, and pregnancy tests for a multitude of animals on a daily or weekly basis. Detection of non-pregnant dariy cattle early after breeding can only improve reproductive performance when together with a operating schedule to quickly present non-pregnant dairy cattle for a subsequent AI service. Accurate detection of non-pregnancy is administered to synchronize estrus or ovulation to reduce the interval to the subsequent AI service.

\section{CONCLUSION}

In conclusion, comprehensive profiling of miRNAs in plasma of pregnant and non-pregnant dairy cattle found specific miRNA expression pattern. Much studies and development efforts are being made toward advancement of a pregnancy diagnosis for dairy cattle. Our study provides an experimental basis to reveal the feasible role of miRNAs as biomarkers in pregnancy diagnosis. This specific pregnancy differentially expressed miRNAs marker can be used as the retrospective detection of early pregnancy biomarkers. Pregnancy-associated microRNA profiling at 8 weeks in bovine was described for the first time and can be used for comparative studies. These miRNAs may have similar function in mammalian species and can be feasible molecular markers for evolution. Coupling a non-pregnancy diagnosis with a management strategies to quickly reinitiate. AI service may improve reproductive performance by decreasing the interval between $\mathrm{AI}$ services and the effectiveness of hormonal ovulation and estrus control protocols initiated at certain physiologic stages post AI breeding. Future experiments are needed in this area to truly understand early identification of pregnancy diagnosis through miRNA biomarkers.

\section{CONFLICTS OF INTEREST}

No potential conflict of interest relevant to this article was reported.

\section{ACKNOWLEDGEMENTS}

This work was carried out with the support of the "Cooperative Research Program for Agriculture Science \& Technology Development (Project title: Development of early pregnancy diagnostic technology using fetal DNA isolated from maternal plasma, Project No. PJ01199401)" Rural Development Administration, Republic of Korea.

\section{AUTHOR CONTRIBUTIONS}

\section{Conceptualization: Hyun-Joo Lim}

Data curation: Hyun-Joo Lim

Formal analysis: Hyun-Joo Lim, Hyun Jong Kim, Ji Hwan Lee Methodology: Hyun-Joo Lim

Writing - original draft: Hyun-Joo Lim

Writing - review \& editing: Hyun-Joo Lim, Hyun Jong Kim, Ji Hwan Lee, Dong Hyun Lim, Jun Kyu Son, Eun-Tae Kim, Gulwon Jang, Dong-Hyeon Kim 


\section{AUTHOR'S POSITION AND ORCID NO.}

HJ Lim, Junior Researcher, https://orcid.org/0000-0001-7059-1553

HJ Kim, Senior Researcher,

https://orcid.org/0000-0001-6217-9374

JH Lee, Junior Researcher,

https://orcid.org/0000-0002-0040-3104

DH Lim, Junior Researcher,

https://orcid.org/0000-0002-8575-0198

JK Son, Junior Researcher,

https://orcid.org/0000-0002-6266-3606

ET Kim, Junior Researcher,

https://orcid.org/0000-0001-7486-5638

G Jang, Senior Researcher,

https://orcid.org/0000-0003-4258-6022

DH Kim, Research Fellow,

https://orcid.org/0000-0003-0756-8419

\section{REFERENCES}

Abdullah M, Mohanty TK, Kumaresan A, Mohanty AK, Madkar AR, Baithalu RK, Bhakat M. 2014. Early pregnancy diagnosis in dairy cattle: economic importance and accuracy of ultrasonography. Adv. Anim. Vet. Sci. 2:464-467.

Anders S, Pyl PT, Huber W. 2015. HTSeq--a Python framework to work with high-throughput sequencing data. Bioinformatics 31:166-169.

Bidarimath M, Khalaj K, Wessels JM, Tayade C. 2014. MicroRNAs, immune cells and pregnancy. Cell. Mol. Immunol. 11:538-547.

Buschmann D, Haberberger A, Kirchner B, Spornraft M, Riedmaier I, Schelling G, Pfaffl MW. 2016. Toward reliable biomarker signatures in the age of liquid biopsies - how to standardize the small RNA-Seq workflow. Nucleic Acids Res. 44:5995-6018.

Cai M, Kolluru GK, Ahmed A. 2017. Small molecule, big prospects: microRNA in pregnancy and its complications. J. Pregnancy 2017:6972732.

Chim SS, Shing TK, Hung EC, Leung TY, Lau TK, Chiu RW, Lo YM. 2008. Detection and characterization of placental microRNAs in maternal plasma. Clin. Chem. 54:482-490.

Cleys ER, Halleran JL, McWhorter E, Hergenreder J, Enriquez VA, da Silveira JC, Bruemmer JE, Winger QA, Bouma GJ. 2014. Identification of microRNAs in exosomes isolated from serum and umbilical cord blood, as well as placentomes of gestational day 90 pregnant sheep. Mol. Reprod. Dev. 81:983-993.

Donker RB, Mouillet JF, Chu T, Hubel CA, Stolz DB, Morelli AE, Sadovsky Y. 2012. The expression profile of C19MC microRNAs in primary human trophoblast cells and exosomes.
Mol. Hum. Reprod. 18:417-424.

Fiandanese N, Viglino A, Strozzi F, Stella A, Williams JL, Lonergan P, Forde N, Iamartino D. 2016. Circulating microRNAs as potential biomarkers of early pregnancy in high-producing dairy cows. Reprod. Fertil. Dev. 28:165.

Gilad S, Meiri E, Yogev Y, Benjamin S, Lebanony D, Yerushalmi N, Benjamin H, Kushnir M, Cholakh H, Melamed N, Bentwich Z, Hod M, Goren Y, Chajut A. 2008. Serum microRNAs are promising novel biomarkers. PLoS One 3:e3148.

Hale BJ, Yang CX, Ross JW. 2014. Small RNA regulation of reproductive function. Mol. Reprod. Dev. 81:148-159.

Häusler SF, Keller A, Chandran PA, Ziegler K, Zipp K, Heuer S, Krockenberger M, Engel JB, Hönig A, Scheffler M, Dietl J, Wischhusen J. 2010. Whole blood-derived miRNA profiles as potential new tools for ovarian cancer screening. Br. J. Cancer 103:693-700.

Hynes RO. 1992. Integrins: versatility, modulation, and signaling in cell adhesion. Cell 69:11-25.

Ioannidis J and Donadeu FX. 2016. Circulating miRNA signatures of early pregnancy in cattle. BMC Genomics 17:184.

Kotlabova K, Doucha J, Hromadnikova I. 2011. Placental-specific microRNA in maternal circulation--identification of appropriate pregnancy-associated microRNAs with diagnostic potential. J. Reprod. Immunol. 89:185-191.

Langmead B and Salzberg SL. 2012. Fast gapped-read alignment with Bowtie 2. Nat. Methods 9:357-359.

Lawrie CH, Gal S, Dunlop HM, Pushkaran B, Liggins AP, Pulford K, Banham AH, Pezzella F, Boultwood J, Wainscoat JS, Hatton CS, Harris AL. 2008. Detection of elevated levels of tumour-associated microRNAs in serum of patients with diffuse large B-cell lymphoma. Br. J. Haematol. 141:672-675.

Luo SS, Ishibashi O, Ishikawa G, Ishikawa T, Katayama A, Mishima T, Takizawa T, Shigihara T, Goto T, Izumi A, Ohkuchi A, Matsubara S, Takeshita T, Takizawa T. 2009. Human villous trophoblasts express and secrete placenta-specific microRNAs into maternal circulation via exosomes. Biol. Reprod. 81:717-729.

Martin M. 2011. Cutadapt removes adapter sequences from high-throughput sequencing reads. EMBnet J. 17:10-12.

Morales-Prieto DM, Ospina-Prieto S, Chaiwangyen W, Schoenleben M, Markert UR. 2013. Pregnancy-associated miRNAclusters. J. Reprod. Immunol. 97:51-61.

Moreira F, Orlandi C, Risco CA, Mattos R, Lopes F, Thatcher WW. 2001. Effects of presynchronization and bovine somatotropin on pregnancy rates to a timed artificial insemination protocol in lactating dairy cows. J. Dairy Sci. 84:16461659.

Navanukraw C, Redmer DA, Reynolds LP, Kirsch JD, GrazulBilska AT, Fricke PM. 2004. A modified presynchronization protocol improves fertility to timed artificial insemination in lactating dairy cows. J. Dairy Sci. 87:1551-1557.

Pohler KG, Green JA, Geary TW, Peres RF, Pereira MH, Vasconcelos JL, Smith MF. 2015. Predicting embryo presence and viability. Adv. Anat. Embryol. Cell Biol. 216:253-270.

Pohler KG, Green JA, Moley LA, Hung W, Hong X, Christenson 
LK, Geary TW, Smith MF. 2015. Circulating microRNAs as biomarkers of early embryonic viability in cattle. In: Proceedings Society for the Study of Reproduction Annual Meeting. San Juan, Puerto Rico. Madison, WI: SSR. (abstract).

Pursley JR, Kosorok MR, Wiltbank MC. 1997. Reproductive management of lactating dairy cows using synchronization of ovulation. J. Dairy Sci. 80:301-306.

Pursley JR, Mee MO, Wiltbank MC. 1995. Synchronization of ovulation in dairy cows using PGF2alpha and GnRH. Theriogenology 44:915-923.

Raposo G and Stoorvogel W. 2013. Extracellular vesicles: exosomes, microvesicles, and friends. J. Cell Biol. 200:373-383.

Reddy KV and Mangale SS. 2003. Integrin receptors: the dynamic modulators of endometrial function. Tissue Cell 35:260-273.

Reese ST, Pereira MC, Vasconcelos JLM, Smith MF, Green JA, Geary TW, Peres RFG, Perry GA, Pohler KG. 2016. Markers of pregnancy: how early can we detect pregnancies in cattle using pregnancy-associated glycoproteins (PAGs) and mi-
croRNAs? Anim. Reprod. 13:200-208.

Reid G, Kirschner MB, van Zandwijk N. 2011. Circulating microRNAs: association with disease and potential use as biomarkers. Crit. Rev. Oncol. Hematol. 80:193-208.

Robinson MD, McCarthy DJ, Smyth GK. 2010. edgeR: a Bioconductor package for differential expression analysis of digital gene expression data. Bioinformatics 26:139-140.

Su L, Zhao S, Zhu M, Yu M. 2010. Differential expression of microRNAs in porcine placentas on days 30 and 90 of gestation. Reprod. Fertil. Dev. 22:1175-1182.

Tesfaye D, Worku D, Rings F, Phatsara C, Tholen E, Schellander K, Hoelker M. 2009. Identification and expression profiling of microRNAs during bovine oocyte maturation using heterologous approach. Mol. Reprod. Dev. 76:665-677.

Tijsen AJ, Creemers EE, Moerland PD, de Windt LJ, van der Wal AC, Kok WE, Pinto YM. 2010. MiR423-5p as a circulating biomarker for heart failure. Circ. Res. 106:1035-1039.

Wang JF, Yu ML, Yu G, Bian JJ, Deng XM, Wan XJ, Zhu KM. 2010. Serum miR-146a and miR-223 as potential new biomarkers for sepsis. Biochem. Biophys. Res. Commun. 394:184-188. 